\title{
Wound Irrigation in Initial Management of Open Fractures
}

TO THE EDITOR: In reporting on the results of the Fluid Lavage of Open Wounds (FLOW) trial, Bhandari et al. (Dec. 31 issue) ${ }^{1}$ describe the initial management of open fracture wounds. According to their protocol, the investigators aimed to perform irrigation and débridement for skin and soft-tissue defects or to close wounds that had delayed union or nonunion with the use of grafting or muscle flaps within 7 to 14 days after the initial surgery. The use of low-pressure wound therapy (vacuum-assisted closure) was described. We wonder whether the investigators adhered to the protocol regarding definitive wound coverage, and we wonder about the number and duration of vacuum-assisted closure procedures used in the trial, since the application of these techniques is controversial.

Delayed wound healing may result in exogenous superinfection, which is often polymicrobial. ${ }^{2}$ In our experience, superinfection is not prevented but may be facilitated by prolonged vacuum-assisted closure therapy. ${ }^{3}$ Skin flora microorganisms can migrate into the wound and adhere to internal fixation material, leading to nonunion or a high rate of recurrent infection. ${ }^{4}$

One of the primary end points of the trial was reoperation at the operative site within 12 months after the initial procedure in order to treat an infection. Information on the number and duration of vacuum-assisted closure procedures used in the trial might provide valuable insight into the association of the use of this procedure with later infection.

Parham Sendi, M.D.
Bern University Hospital
Bern, Switzerland
parham.sendi@ifik.unibe.ch
Martin A. McNally, M.D.
Oxford University Hospitals
Oxford, United Kingdom
$\quad$ No potential conflict of interest relevant to this letter was re-
ported.
1. The FLOW Investigators. A trial of wound irrigation in the
initial management of open fracture wounds. N Engl J Med
2015;373:2629-41.
2. Zimmerli W, Sendi P. Internal fixation-associated infection.
In: Bennett JE, Dolin R, Blaser MJ, eds. Mandell, Douglas, and
Bennett's principles and practice of infectious diseases. 8th ed.
Philadelphia: Saunders, 2015:1335-8.
3. Olesen UK, Juul R, Bonde CT, et al. A review of forty five

open tibial fractures covered with free flaps: analysis of complications, microbiology and prognostic factors. Int Orthop 2015; 39:1159-66.

4. Diefenbeck M, Mennenga U, Gückel P, Tiemann AH, Mückley T, Hofmann GO. Vacuum-assisted closure therapy for the treatment of acute postoperative osteomyelitis. Z Orthop Unfall 2011;149:336-41. (In German.)

DOI: 10.1056/NEJMc1601157

TO THE EDITOR: Bhandari et al. analyzed the effectiveness of irrigation in the management of open fracture wounds and found no difference in the primary trial end-point event (reoperation within 12 months after the index procedure in order to treat an infection, manage a woundhealing problem, or promote bone healing) according to the type of irrigation pressure used. However, the rate of reoperation was higher in the soap group than in the saline group.

Even if large-volume irrigation is always recommended, débridement is the most important procedure to perform. Adequate débridement is thought to be key to reducing the prevalence of infection after open fracture. ${ }^{1-3}$ However, information regarding débridement practices is lacking in the protocol for the FLOW trial. How was the quality of the initial débridement evaluated by surgeons? Débridement should be extensive in patients with Gustilo-Anderson type III fractures but limited in patients with Gustilo-Anderson type I fractures (the Gustilo-Anderson classification ranges from type I to type III, with higher numbers indicating greater injury and a higher potential for complications). ${ }^{4}$ Culture specimens should be obtained from the wound after initial débridement, as well as before and after irrigation at each operative procedure, in order to assess the reduction of the bacteriologic load according to the type of irrigation pressure used.

\section{Olivier Barbier, M.D.}

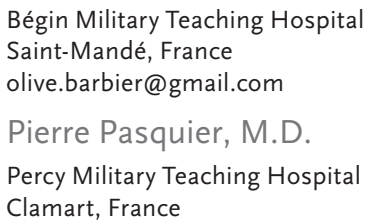

No potential conflict of interest relevant to this letter was reported. 
1. Anglen JO. Comparison of soap and antibiotic solutions for irrigation of lower-limb open fracture wounds: a prospective, randomized study. J Bone Joint Surg Am 2005;87:1415-22.

2. Seng VS, Masquelet AC. Management of civilian ballistic fractures. Orthop Traumatol Surg Res 2013;99:953-8.

3. Barbier O, Malgras B, Pasquier P, Rigal S. Training for treating open fractures in the austere setting. Int Orthop 2014;38: 2215-6.

4. Sathiyakumar V, Thakore RV, Stinner DJ, Obremskey WT, Ficke JR, Sethi MK. Gunshot-induced fractures of the extremities: a review of antibiotic and debridement practices. Curr Rev Musculoskelet Med 2015;8:276-89.

DOI: $10.1056 /$ NEJMc1601157

THE AUTHORS REPLY: Sendi and McNally inquire about vacuum-assisted closure of wounds in the FLOW trial. A post hoc analysis indicated that 331 patients underwent a vacuum-assisted closure in our trial. Although we did not stratify patients according to the use or nonuse of vacuum-assisted closure, the study groups (the irrigation-pressure groups and the solution groups) were balanced with respect to the use of the vacuum-assisted technique.

As expected, the use of vacuum-assisted closure was directly proportional to the severity of the open fracture wound (according to the Gustilo-Anderson fracture type). In all types of fracture wounds, the use of vacuum-assisted wound closure was associated with an increased rate of infection (by a factor of 5.3 among patients with type I wounds, a factor of 2.4 among patients with type II wounds, a factor of 2.0 among patients with type IIIA wounds, and a factor of 1.5 among patients with type IIIB wounds) (Table 1).

A new analysis of factors associated with rates of reoperation is currently a priority for the prespecified secondary analysis of our data set. This analysis of factors includes wound closure and management as a covariate among several other variables. The extent to which vacuum-assisted wound closure predicts infection within 1 year after fracture may be of interest in future trials. Exploration of the biologic rationale for this observation is warranted.

In reply to Barbier and Pasquier: our trial carefully evaluated approaches to wound irrigation as procedures included in the composite primary end point. However, removal of all nonviable skin, muscle, and other tissues, also called wound débridement, was standardized to the extent feasible in our global pragmatic trial. Although the approach to débridement was left to the discretion of the operating surgeon, we encouraged a thorough approach consistent with the standard of care. Indeed, similar amounts of skin, muscle, fascia, and bone were débrided in patients across the treatment groups; as expected, these amounts increased with the severity of fracture wounds. Furthermore, our trial did stratify patients according to the severity of open wounds to ensure a balance across treatment groups with respect to the most severe types of wounds.

\begin{tabular}{|c|c|c|c|c|c|}
\hline \multirow[t]{3}{*}{$\begin{array}{c}\text { Gustilo-Anderson } \\
\text { Type' }\end{array}$} & \multicolumn{2}{|c|}{ Vacuum-Assisted Closure } & \multicolumn{2}{|c|}{ No Vacuum-Assisted Closure } & \multirow[t]{3}{*}{ P Value } \\
\hline & Infection & No Infection & Infection & No Infection & \\
\hline & \multicolumn{2}{|c|}{ no. of patients/total no. (\%) } & \multicolumn{2}{|c|}{ no. of patients/total no. (\%) } & \\
\hline I & $3 / 10(30.0)$ & $7 / 10(70.0)$ & $36 / 629(5.7)$ & $593 / 629(94.3)$ & NA \\
\hline II & $17 / 62(27.4)$ & $45 / 62(72.6)$ & $93 / 834(11.2)$ & $741 / 834(88.8)$ & $<0.001$ \\
\hline IIIA & $37 / 130(28.5)$ & $93 / 130(71.5)$ & $76 / 519(14.6)$ & $443 / 519(85.4)$ & $<0.001$ \\
\hline IIIB & $41 / 130(31.5)$ & $88 / 130(67.7)$ & $22 / 103(21.4)$ & $81 / 103(78.6)$ & $<0.001$ \\
\hline
\end{tabular}

* NA denotes not applicable.

$\uparrow$ The Gustilo-Anderson classification ranges from type I to type III, with higher numbers indicating greater injury and a higher potential for complications.

The comparisons of the use or nonuse of vacuum-assisted closure of wounds and fracture type with the rate of infections were analyzed with the use of multivariable binary logistic regression. $\mathrm{P}<0.001$ for the comparison of vacuumassisted closure of wounds with no vacuum-assisted closure (odds ratio, 2.39; $95 \%$ confidence interval, 1.76 to 3.26 ) with adjustment for Gustilo-Anderson fracture type. 
Mohit Bhandari, M.D., Ph.D.

Brad A. Petrisor, M.D.

McMaster University

Hamilton, ON, Canada

bhandam@mcmaster.ca
Kyle J. Jeray, M.D.

Greenville Health System

Greenville, SC

Since publication of their article, the authors report no further potential conflict of interest.

DOI: 10.1056/NEJMc1601157

\section{Crizotinib Resensitization by Compound Mutation}

TO THE EDITOR: Shaw et al. (Jan. 7 issue) ${ }^{1}$ report a case of anaplastic lymphoma kinase (ALK)-positive non-small-cell lung cancer that was successfully re-treated with crizotinib; in their report, the authors show the importance of repeat biopsy in patients with lung cancer treated with targeted therapies. In fact, the expansion of a double ALK mutant clone (p.C1156Y-p.L1198F) led to a restored sensitivity to crizotinib that resulted in a dramatic response after the disease had progressed during ceritinib and lorlatinib therapies. However, because of unreachable sites of disease, not many patients can undergo repeat biopsies. Circulating cell-free DNA could represent a useful source for molecular profiling in patients with ALK-positive non-small-cell lung cancer, as it does in patients with epidermal growth factor receptor (EGFR)-mutated non-small-cell lung cancer. ${ }^{2}$ We monitored plasma DNA before and during therapy with brigatinib (another ALK inhibitor) in patients who had tumors that had progressed during treatment with crizotinib; we isolated the mutation p.L1196M alone in one patient and p.L1196M associated with p.G1269A in another patient. The DNA levels of both mutations decreased during therapy, which corresponded to excellent radiologic responses. A third patient had an ALK p.F1174L mutation detected in circulating cell-free DNA after 2 months, but the patient has not yet had disease progression. In conclusion, molecular profiling of ALK-positive tumors is crucial, and circulating cell-free DNA represents a valid source to monitor.

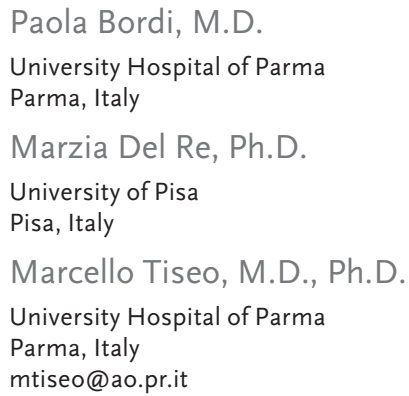

No potential conflict of interest relevant to this letter was reported.

1. Shaw AT, Friboulet L, Leshchiner I, et al. Resensitization to crizotinib by the lorlatinib ALK resistance mutation L1198F. N Engl J Med 2016;374:54-61.

2. Bordi P, Del Re M, Danesi R, Tiseo M. Circulating DNA in diagnosis and monitoring EGFR gene mutations in advanced non-small cell lung cancer. Transl Lung Cancer Res 2015;4:58497.

DOI: $10.1056 /$ NEJMc1601366

THE AUTHORS REPLY: In our case report, we studied the evolution of resistance to multiple, different ALK-targeted therapies using serial tumor biopsies. We agree that repeat biopsies are not always practical or technically feasible and that circulating cell-free DNA holds great promise of real-time, noninvasive monitoring of genetic resistance mechanisms. Although the resistant cancer in our patient was remarkably homogeneous, cancers in other patients can show considerable heterogeneity at the time of resistance. ${ }^{1-3}$ In patients with heterogeneous cancer, repeat biopsies may provide limited information because only a single site of disease is sampled. In contrast, circulating cell-free DNA can theoretically identify genetic resistance mechanisms across multiple sites of disease and thus may be particularly helpful in evaluating the scope and effect of tumor heterogeneity. Future clinical studies may help to establish the effectiveness of emerging cell-free DNA technologies in identifying clinically relevant mechanisms of resistance.

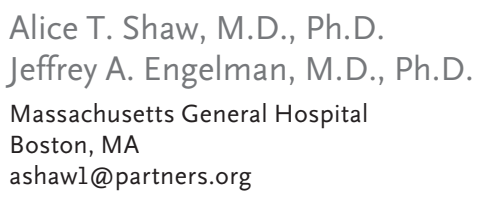

Since publication of their article, the authors report no further potential conflict of interest.

1. Crystal AS, Shaw AT, Sequist LV, et al. Patient-derived models of acquired resistance can identify effective drug combinations for cancer. Science 2014;346:1480-6.

2. Niederst MJ, Sequist LV, Poirier JT, et al. RB loss in resistant 